\title{
HEART RATE DETECTION USING HILBERT TRANSFORM
}

\author{
S. Thulasi Prasad ${ }^{1}$, Dr. S. Varadarajan ${ }^{2}$ \\ ${ }^{1}$ Associate Professor, Dept. of ECE, CVSE, Andhra Pradesh, India, stprasad123@yahoo.co.in \\ ${ }^{2}$ Professor, Dept. of ECE, SVUCE, Andhra Pradesh, India, varadasouri@gmail.com
}

\begin{abstract}
The electrocardiogram (ECG) is a well known method that can be used to measure Heart Rate Variability (HRV). This paper describes a procedure for processing electrocardiogram signals (ECG) to detect Heart Rate Variability (HRV). In recent years, there have been wide-ranging studies on Heart rate variability in ECG signals and analysis of Respiratory Sinus Arrhythmia (RSA). Normally the Heart rate variability is studied based on cycle length variability, heart period variability, RR variability and RR interval tachogram. The HRV provides information about the sympathetic-parasympathetic autonomic stability and consequently about the risk of unpredicted cardiac death. The heart beats in ECG signal are detected by detecting R-Peaks in ECG signals and used to determine useful information about the various cardiac abnormalities. The temporal locations of the $R$-wave are identified as the locations of the QRS complexes. In the presence of poor signal-to-noise ratios or pathological signals and wrong placement of ECG electrodes, the QRS complex may be missed or falsely detected and may lead to poor results in calculating heart beat in turn inter-beat intervals. We have studied the effects of number of common elements of QRS detection methods using MIT/BIH arrhythmia database and devised a simple and effective method. In this method, first the ECG signal is preprocessed using band-pass filter; later the Hilbert Transform is applied on filtered ECG signal to enhance the presence of QRS complexes, to detect R-Peaks by setting a threshold and finally the RR-intervals are calculated to determine Heart Rate. We have implemented our method using MATLAB on ECG signal which is obtained from MIT/BIH arrhythmia database. Our MATLAB implementation results in the detection of QRS complexes in ECG signal, locate the R-Peaks, computes Heart Rate (HR) by calculating RR-internal and plotting of HR signal to show the information about HRV.
\end{abstract}

Index Terms: ECG, QRS complex, R-Peaks, HRV, Heart Rate signal, RSA, Hilbert Transform, Arrhythmia, MIT/BIH, MATLAB and Lynn's filters

\section{INTRODUCTION}

The World Health Organization has discovered a fact that the most frequent cause of deaths worldwide is due to cardiac arrest [1]. Hence a strong focus has been laid on preventative, medicinal, and technological advances on cardiac health research which in turn made leading researchers to work on improving the conventional cardiovascular-diagnosis technologies used in hospitals, clinics and the home. The ECG analysis is most common clinical cardiac test used for screening various cardiac abnormalities. Therefore, the analysis of ECG signals has been extensively investigated over the past two decades using Digital Signal Processing [2][3].

A graphical record of bioelectrical signal generated by the human heart during the cardiac cycle [4] is called as ECG (Electro Cardio Gram). The electrocardiogram permits us to determine many electrical and mechanical defects of the heart such as the heart rate and other cardiac parameters regarding Auricular and ventricular hypertrophy, Myocardial Infarction (heart attack), Arrhythmias, Pericarditis, Generalized suffering affecting heart and blood pressure. The main parts of ECG waveform are the $\mathrm{P}$ wave, PR interval, QRS complex, ST segment, T wave and QT interval which represents polarization of atria and ventricles in a sequential manner. These parts are shown in the Fig-1. The frequency spectrum of ECG signal ranges from $0.05 \mathrm{~Hz}$ to $100 \mathrm{~Hz}$ with QRS complexes concentrating around $10 \mathrm{~Hz}$. The power spectrum of ECG signal [5][6] is shown in the Fig-2. In addition to the ECG wave the noise due to power line interferences of $50-\mathrm{Hz}$, EMG from muscles, motion artifact from the electrode and skin interface, and possibly other interference from electro-surgical equipment in the operating room is also picked up by electrodes, when the ECG signal is recorded. Fig-3 shows the baseline Wander due of Sudden Body Movements by elevating the effected part of the ECG signal. Analysis of ECG signals requires extracting the signal of interest, the QRS complex, from the noise sources and the $\mathrm{P}$ and $\mathrm{T}$ waves. 


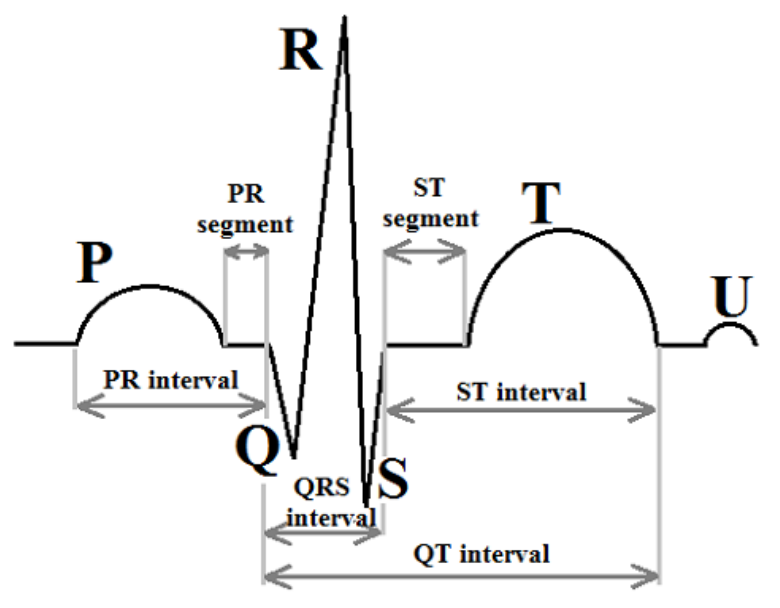

Fig-1: Typical ECG signal with specified waves, intervals and segments

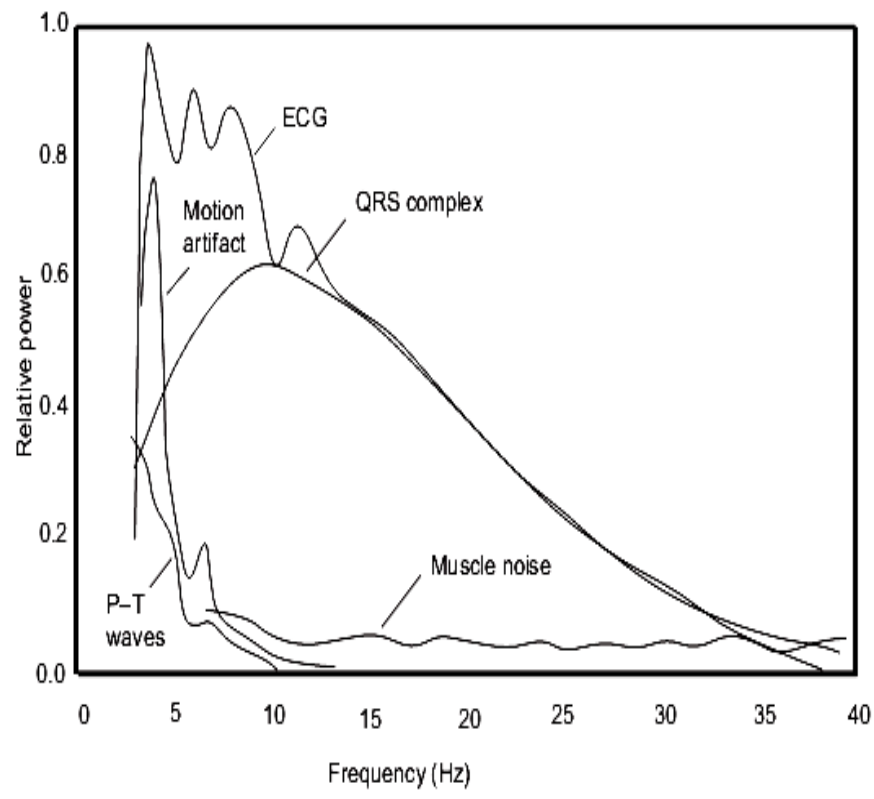

Fig-2: Power spectrum of the $P$ \& $T$ waves, QRS complex, Motion artifact and muscle noise.

The classic definition of a wave boundary is the time instant at which the wave crosses a certain amplitude threshold level. Unfortunately, this definition is not well-suited for the common situation when the ECG contains baseline wander; therefore this definition is rarely applied in practice. Instead, many methods for wave delineation exploit the change in slope that occurs at a boundary to avoid the problems because of lowfrequency noise

In recent years using of microcomputers to process the ECG signal has increased and giving efficient results. In fact, many systems have already been designed and implemented to perform signal processing tasks such as12-lead offline ECG analysis, Holter tape analysis, and real-time patient monitoring. All these applications require an accurate detection of the QRS complex of the ECG. The earliest method used to detect QRS complexes and R-waves in ECG signals are based on the first and second order derivative of the ECG. This method is characterized by low computational complexity and results in relatively poor detection in the presence of noise due to baseline drift, noise and artifacts, as well as changes in the QRS morphology. Recently several methods have been developed using the field of linear and nonlinear filtering, wavelet transform, artificial neural networks, genetic algorithms and linear prediction. All these algorithms are generally much more complex compared to derivative-based method.

In this paper, a method is proposed that simultaneously meets the demands of a low computational load and a high detection performance. PhysioNet provides a large set of ECG records for use in the Challenge. Therefore, the next step is analyzing the acceptable ECG signal for diagnosis without relying on an expert for interpretation.

\section{PRE-PROCESSING}

ECG signal can be analyzed and processed in two domains, time and frequency [7]. We have chosen frequency domain and used Hilbert Transform to analyze ECG signal. Hilbert Transform is usually used to rectify the phase in order to create a signal with outstanding peaks in the location of the $\mathrm{R}$ peaks [8]. To use Hilbert transform, first the ECG signal is band-pass filtered, then Hilbert transformed, followed by threshold detection.

The pre-processing of ECE signal involves removing of baseline wanders and power line interference using band pass filter. The baseline wander is usually ranges below $0.5 \mathrm{~Hz}$ under normal conditions and extends up to $0.75 \mathrm{~Hz}$ with movement of the body and during the stress. The changes in beat morphology that are not originating from cardiac system can be reduced by removing baseline wander. With the Removal of baseline wander analysis of low frequency ST segment to diagnose ischemia becomes easy. The baseline wander is removed with the help of a linear time-invariant high-pass filter designed choosing cut-off frequency of $0.5 \mathrm{~Hz}$, considering the slowest heart rate, since the heart beat during bradycardia may be around 40 beats/ minute (approximately 0.6 $\mathrm{Hz}$ ). 


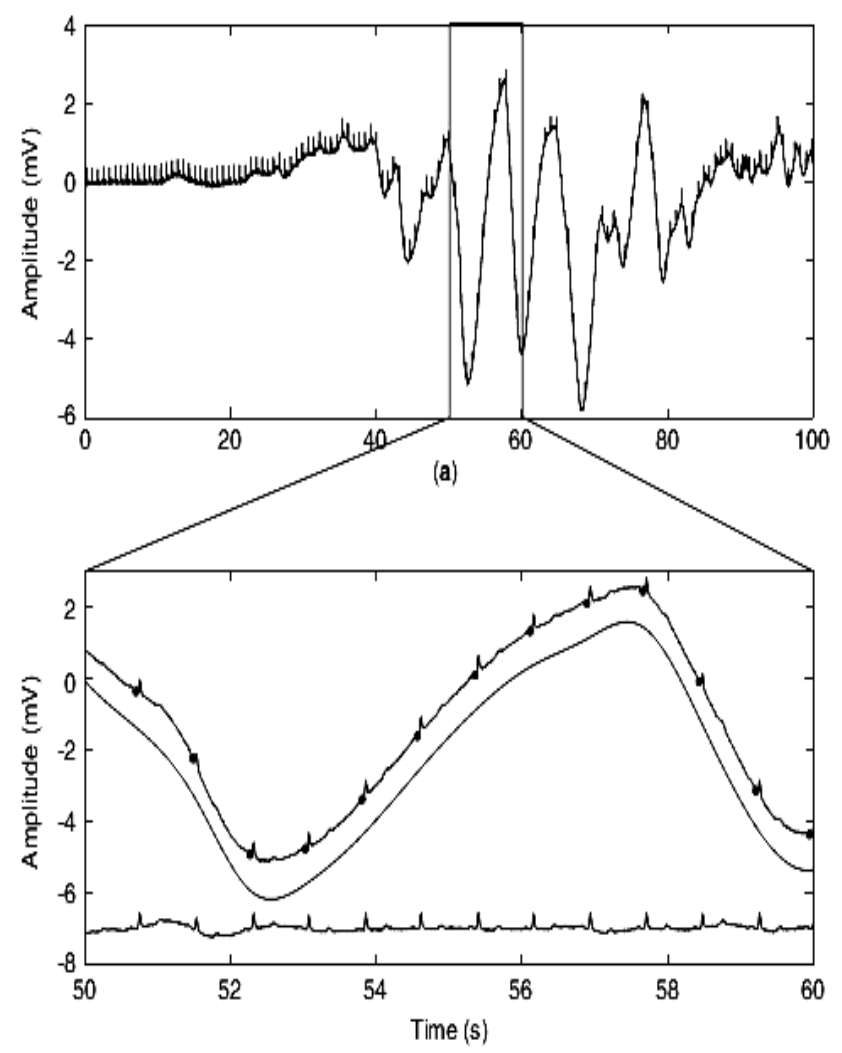

(b)

Fig-3: Baseline Wander due of Sudden Body Movements

Another source of noise is due electromagnetic fields caused by a power line at $50 \mathrm{~Hz}$. This noise makes the analysis of ECG signals more difficult as the delineation of low-amplitude waveforms becomes unreliable and spurious waveforms may be introduced. A band rejection filter is used to overcome the effect of power line interference. The digital low-pass and highpass filters can be used to realize ban-pass filter [9]. These filters are implemented by Lynn's filters and successfully applied on various low frequency signals [10]. Now the same filter technology can be used to design band-pass filter to remove baseline wander

\subsection{Low-Pass Filter}

The low-pass filter designed by Lynn is represented in simple and effective form with the following transfer function [11].

$$
\begin{aligned}
H_{1}(z) & =\frac{\left(1-z^{-\beta}\right)^{2}}{\left(1-z^{-1}\right)^{2}} \\
H_{1}(\omega) & =\frac{\left(1-e^{-j \beta \omega}\right)^{2}}{\left(1-e^{-j \omega}\right)^{2}}
\end{aligned}
$$

Amplitude response of low-pass filter is obtained as follows

$$
\left|H_{1}(\omega)\right|=\frac{\left|\sin ^{2}\left(\frac{\beta \omega}{2}\right)\right|}{\left|\sin ^{2}\left(\frac{\omega}{2}\right)\right|}
$$

And the corresponding difference equation

$$
\mathrm{y}(\mathrm{n})=2 \mathrm{y}(\mathrm{n}-1)-\mathrm{y}(\mathrm{n}-2)+\mathrm{x}(\mathrm{n})-2 \mathrm{x}(\mathrm{n}-\beta)+\mathrm{x}(\mathrm{n}-2 \beta)
$$

Value of $\beta$ is chosen depending upon $3 \mathrm{~dB}$ cut off frequency. For example if the cut off frequency is $35 \mathrm{~Hz}$ and sampling frequency is $430 \mathrm{~Hz}$, then $\beta$ is 4 and the corresponding difference equation is as follows

$$
y(n)=2 y(n-1)-y(n-2)+x(n)-2 x(n-4)+x(n-8)
$$

\subsection{High-Pass Filter}

Similar to low-pass filter, Lynn's high-pass [11] is designed by subtracting a low pass filter from an all pass filter with delay. The transfer function of the low pass filter is

$$
\begin{aligned}
H_{2}(z) & =\frac{\left(1-z^{-\beta}\right)}{\left(1-z^{-1}\right)} \\
H_{2}(\omega) & =\frac{\left(1-e^{-j \beta \omega}\right)}{\left(1-e^{-j \omega}\right)}
\end{aligned}
$$

Amplitude response of high-pass filter is obtained as follows

$$
\left|H_{2}(\omega)\right|=\frac{\left|\sin \left(\frac{\beta \omega}{2}\right)\right|}{\left|\sin \left(\frac{\omega}{2}\right)\right|}
$$

The filter's group delay is $(\beta-1) / 2$ and the associated gain for $\omega=0$ is $\beta$ determined evaluating $|\mathrm{H}(\omega=0)|$.

Now the high-pass filter transfer function is obtained as follows

$$
H_{3}(z)=z^{\frac{-(\beta-1)}{2}}-\frac{\left(1-z^{-\beta}\right)}{\beta\left(1-z^{-1}\right)}
$$

And corresponding difference equation is 


$$
\begin{array}{r}
y(n)=\frac{y(n-1)-x(n)}{\beta}+x\left(n-\frac{(\beta-1)}{2}\right) \\
-x\left(n-\frac{(\beta-1)}{2}-1\right)+\frac{x(n-\beta)}{\beta}
\end{array}
$$

For sampling frequency of $430 \mathrm{~Hz}$ and cut off frequency of 0.5 $\mathrm{Hz}$ the value of $\beta$ is found to be 33

$$
y(n)=0.0303[y(n-1)-x(n)+x(n-33)]+x(n-16)-x(n-15)
$$

The original ECG signal, only low-pass, only high-pass and band-pass filtered signals are shown plotted in Fig-4 using MATLAB
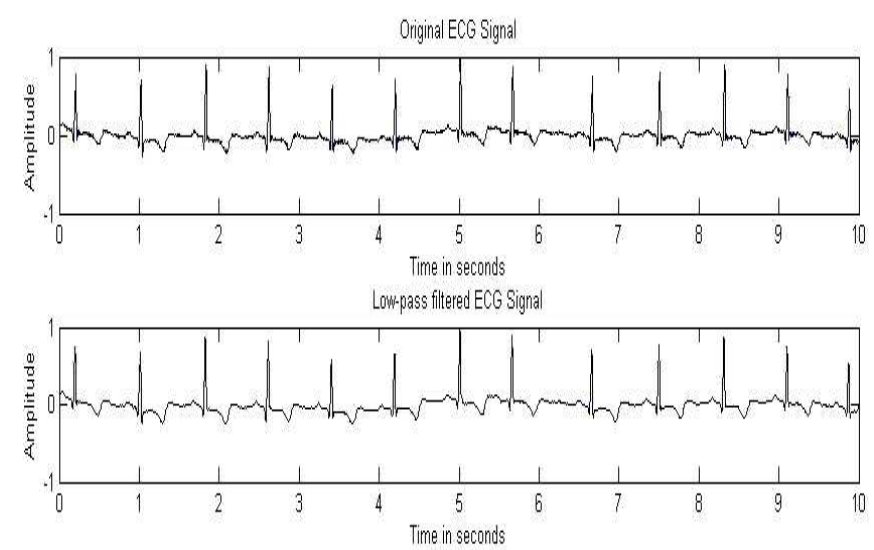

High pass fittered ECG Signal

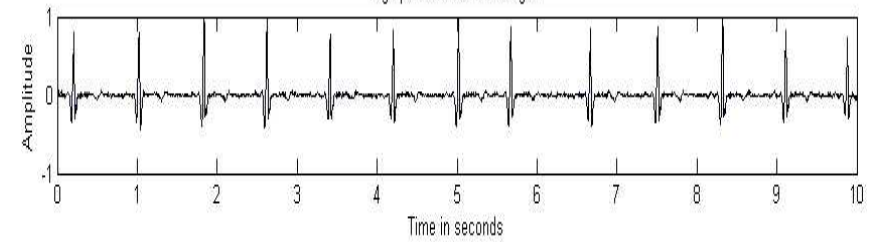

Band pass filtered ECG Signal

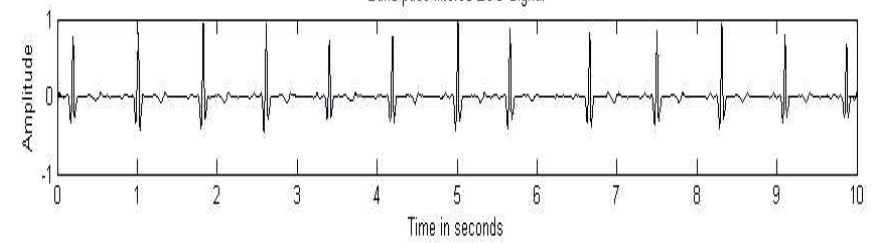

Fig- 4: Original ECG and Pre-processed Signals

\section{HEART RATE DETECTION}

To find heart rate (HR), detection of QRS complex, in turn identification of R-Peaks is essential. Any automated QRS detection algorithm structure involves QRS enhancement and QRS detection [12]. The QRS enhancement stage is used to enlarge the QRS complex compared to the other ECG features ( $\mathrm{P}, \mathrm{T}$, and noise). The QRS enhancement stage is occasionally called pre-processing or feature extraction, which we have done already under preprocessing section. If the R-Peak is required to be detected, an extra step is needed to determine the maximum amplitude value within the detected QRS complex.

Since R-wave is positive waveform and highest peak in ECG signal, the time interval between two successive R-wave peaks is used to calculate HR (beats/minute) as follows [13].

$$
H R=\frac{60}{R R-I N T E R V A L} \text { beats/minute }
$$

Hilbert transform is used to identify the R-wave peaks. Hilbert transform of a real signal is defined as

$$
\begin{aligned}
x_{h}(t) & =\frac{1}{\pi} \int_{-\infty}^{\infty} \frac{x(\tau)}{t-\tau} d \tau \\
& =x(\tau) * \frac{1}{\pi t}
\end{aligned}
$$

And the envelop $x_{e}(n)$ of ECG signal, $x(n)$, is as

$$
\begin{aligned}
x_{e}(n) & =\sqrt{x^{2}(n)+x_{h}^{2}(n)} \\
& \approx|x(n)|+\left|x_{h}(n)\right|
\end{aligned}
$$

After the ECG signal is filtered in a band-pass filter, the envelop of this ECG signal is computed with help of the above equation and shown plotted in the Fig-4. Here the polarity problem of ECG signal caused by wrong placement of electrodes is also solved by Hilbert Transform [14].

Using the following algorithm R-Peaks from the Hilbert Transformed ECG signal are located and separation between consecutive R-Peaks are calculated.

\section{$\%$ ALGORITHM TO APPLY HILBERT TRANSFORM $\%$ AND DETERMINE R-PEAKS}

\section{$\%$ HILBER TRANSFORM}

$\mathrm{s}=\mathrm{x} 3 ; \%$ Band-pass filtered ECG signal

$\mathrm{d}=1 /($ pi*length $(\mathrm{s}))$;

$\mathrm{s} 2=\operatorname{conv}(\mathrm{s}, \mathrm{d})$

$\mathrm{xe}=\operatorname{abs}(\mathrm{s})+\operatorname{abs}(\mathrm{s} 2) ; \%$ Envelop $\mathrm{x}_{\mathrm{e}}(\mathrm{t})$

\section{\% MOVING WINDOW INTEGRATION}

$\%$ MAKE IMPULSE RESPONSE

$\mathrm{h}=$ ones $(1,31) / 31$;

Delay $=15 ; \%$ Delay in samples

$\%$ Apply filter

$\mathrm{x} 6=\operatorname{conv}(\mathrm{xe}, \mathrm{h})$;

$\mathrm{x} 6=\mathrm{x} 6(15+[1: \mathrm{N}])$; 
$x 6=x 6 / \max (\operatorname{abs}(x 6))$

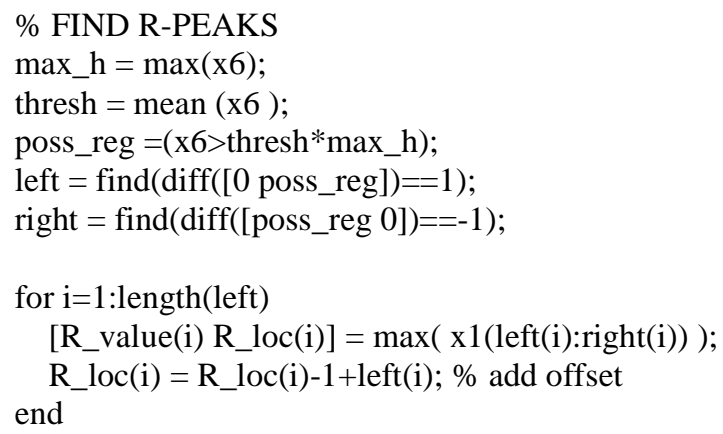

R_loc $=R \_l o c\left(\right.$ find $\left(R \_\right.$value $\left.\left.>0\right)\right)$

$R \_$value $=R$ _value (find $\left(R \_v a l u e>0\right)$ )

beats $=$ length $\left(\mathrm{R} \_\right.$loc $)$

time $=0: 1 / \mathrm{fs}:(\text { length }(\mathrm{x} 1)-1)^{*} 1 / \mathrm{fs}$;

\section{$\%$ CALCULATE THE HEARTRATE}

beat_frequency $=($ length $(\mathrm{x} 1)-1) * 1 / \mathrm{fs} /$ beats

$\mathrm{bpm}=$ beat_frequency*60

$\mathrm{HR}=60 . / \mathrm{diff}\left(\mathrm{R} \_l o c\right) * \mathrm{fs}$

\section{\% PLOTING RESULTS}

figure

subplot $(3,1,1)$

plot $(\mathrm{t}, \mathrm{x} 1 / \max (\mathrm{x} 1))$;

title('Orignal ECG Signal');

xlabel('Time in seconds');

ylabel('Amplitude');

subplot $(3,1,2)$

plot (t,x3/max (x3), t(R_loc) ,R_value , ' $\left.\mathrm{r}^{\wedge}\right)$;

legend('ECG','R','S','Q');

title('ECG Signal with R points');

xlabel('Time in seconds');

ylabel('Amplitude');

$\% x \lim \left(\left[\begin{array}{ll}1 & 6\end{array}\right]\right)$

$\operatorname{subplot}(3,1,3)$

stairs(HR)

title('Heart Rate Signal of ECG ');

xlabel('Time in seconds');

ylabel('HR $\left.\left(\mathrm{min}^{-1}\right)^{\prime}\right)$;

$x \lim \left(\left[\begin{array}{ll}0 & 10\end{array}\right]\right)$

\section{RESULTS}

We tested our procedure on the ECG signal that is obtained from MIT/BIH database. According to the algorithm, first the signal is low-pass filtered and then high-pass filtered to remove noise due to baseline wander, other physiological signals, to attenuate the low frequencies characteristics of $\mathrm{P}$ and $\mathrm{T}$ waves, to isolate and also enhance the predominant QRS energy centered at $10 \mathrm{~Hz}$
The filtered ECG signal is processed with Hilbert Transform to distinguish the QRS complexes from other parts of ECG wave. Next The QRS complex is detected by the determination of the temporal location of the R-wave with a combined maximum/minimum search. A simple decision logic decides whether to use the maximum or the minimum position of the search as the temporal location of the R-wave. Once the RPeaks are located Heart Rate signal is determined from the separation between consecutive R-Peaks and plotted. Fig-4 shows the Original ECG signal, ECG signal with R-Peaks and the corresponding Heart Rate signal as function of time
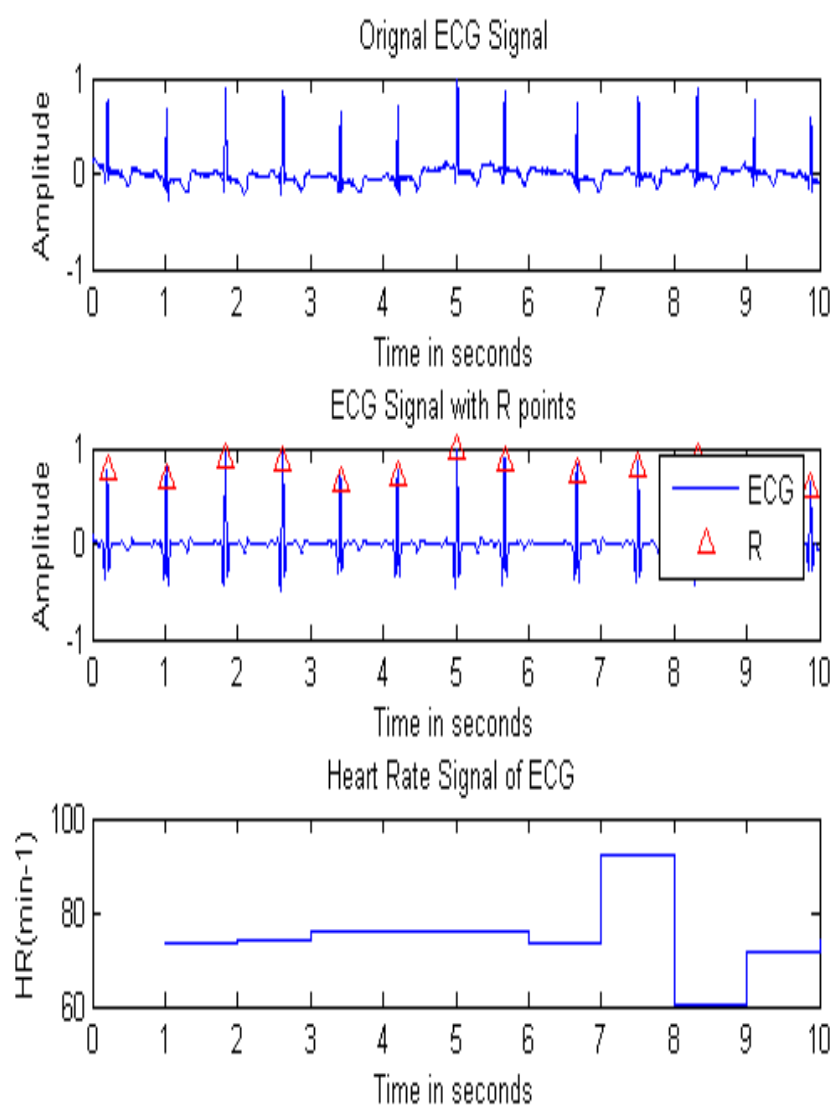

Fig-4: Original ECG signal, ECG signal with R-Peaks and Heart Rate signal

\section{CONCLUSIONS}

Our work is a good demonstration of the potential application of Software to Hardware, especially in the field of biotechnology. It is possible to provide a feedback control to slowdown or speed up breathing rate to resonant frequency where the amplitude of HRV is maximized to maintain the same breathing frequency. The likely use of this work in biomedical signal processing show the validity of the affirmation in academic and professional aspects in medical field 


\section{REFERENCES}

[1]. Global Cardiovascular InfoBase, Ottawa, Canada: WHO Collaborating Centre on Surveillance of Cardiovascular Diseases, Surveillance and Risk Assessment Division, CCDPC, Public Health Agency of Canada and The Ottawa Hospital, University of Ottawa; [updated: 2006 March 3; cited: 2010 May 15].

[2]. A DSP Practical Application: Working on ECG Signal, Applications of Digital Signal Processing, Dr. Christian Cuadrado-Laborde (Ed.), ISBN: 978-953-307-406-1.

[3]. D. Lindner, (January 2009). Introduction to Signals and Systems: Mc Graw Hill Company, First Edition, ISBN 978025-6252-590, USA.

[4]. N. Goldschlager, (June 1989). Principles of Clinical Electrocardiographic: Appleton \& Lange, 13th edition, ISBN 978-083-8579-510, Connecticut, USA.

[5]. C. Vidal, P. Charnay, P. Arce, (2008). Enhancement of a QRS Detection Algorithm Based on the First Derivative Using Techniques of a QRS Detector Algorithm Based on Non-Linear Transformation, Proceedings of IFMBE 2008, 4th European Conference of the International Federation for Medical and Biological Engineering, Volume 22, Part 6, pp. 393-396, ISBN 978-354-0892-076, Antwerp, Belgium, December 2009.

[6]. C. Vidal, V. Gatica, (2010), Design and Implementation of a Digital Electrocardiographic System, University of Antioquia Engineering Faculty Scientific Magazine, No. 55, (September 2010), pp. 99-107, ISSN 0120-0230, Antioquia, Colombia.

[7]. J. Proakis, D. Manolakis, (2007). Digital Signal Processing: Principles, Algorithms, and Applications, Prentice Hall, 3rd edition, ISBN 978-013-3737-622, New Jersey, USA.

[8]. N. Arzeno, Z. Deng, and C. Poon, Analysis of First Derivative Based QRS Detection Algorithms. IEEE Transactions on Biomedical Engineering, 2008; 55(2): 478484.

[9]. J. Pan, and W. J. Tompkins, A Real-Time QRS Detection Algorithm. IEEE Transactions on Biomedical Engineering, 1985; 32(3): 230-236.

[10]. M. L. Ahlstrom, W. J. Tompkins, (1985). Digital Filters for Real-Time ECG Signal Processing Using Microprocessors, IEEE Transaction on Biomedical Engineering, Vol.32, No.9, (March 2007), pp. 708-713, ISSN 0018-9294.

[11]. P. S. Hamilton, W. J. Tompkins, (1986). Quantitative Investigation of QRS Detection Rules Using MIT/BIH Arrhythmia Database, IEEE Transactions on Biomedical Engineering, Vol.31, No.3, (March 2007), pp. 1157-1165, ISSN 0018-9294.

[12]. Y. H. Hu, W. J. Tompkins, J. L. Urrusti, and V. X. Afonso, Applications of artificial neural networks for ECG signal detection and classification. Electrocardiology, 1993; 26 (Suppl.): 66-73.

[13]. J. Parák, and J. Havlík, ECG Signal Processing and Heart Rate Frequency Detection Methods. In Proceedings of Technical Computing Prague, 2011; 8.11.2011
[14]. J. Pucik, J. Cocherova, Elena: Bio-signal analysis STU Publishing House Bratislava, 2008, 121, pp-ISBN 978-80-2272833-1.

\section{BIOGRAPHIES}

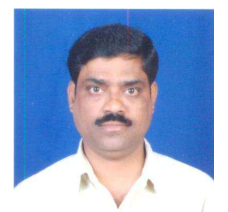

S. Thulasi Prasad received the M. Tech degree in Electronics and Communication Engineering from S.V.University College of Engineering, Tirupati, in 2003. He is presently working as Associate Professor in the Dept. of ECE in CVSE.

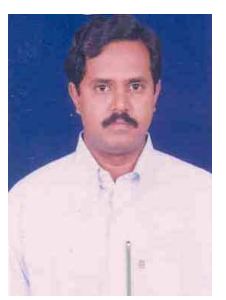

Dr. S. Varadarajan received the M. Tech degree in Electronics and Communication Engineering from NIT, Warangal and Ph.D degree from S.V.University College of Engineering, Tirupati. He is presently working as Professor in the Dept. of ECE in S.V.University College of Engineering 